\title{
On inverse problem for a class of fourth order strongly damped wave equations
}

\author{
Nam Danh Hua Quoc ${ }^{1, *}$, Can Nguyen Huu ${ }^{2, * *}, A u$ Vo Van ${ }^{3, * * *}$, and Binh Tran Thanh ${ }^{4, * * * *}$ \\ ${ }^{1}$ Department of Scientific Research Management, Thu Dau Mot University, Thu Dau Mot City, Binh \\ Duong Province, Viet Nam \\ ${ }^{2}$ Applied Analysis Research Group, Faculty of Mathematics and Statistics, Ton Duc Thang University, \\ Ho Chi Minh City, Viet Nam \\ ${ }^{3}$ Faculty of General Sciences, Can Tho University of Technology, Can Tho City, Viet Nam \\ ${ }^{4}$ Faculty of Applied Mathematics, Sai Gon University, Ho Chi Minh City, Viet Nam
}

\begin{abstract}
In this paper, we study the initial inverse problem for a class of fourth order strongly damped linear wave equations. In the beginning, we show that the problem is ill-posed in the sense of Hadamard. Next, we propose the method called: the Fourier truncation method for stabilizing the problem. Convergence estimates are established under a priori regularity assumptions on the problem data.
\end{abstract}

Mathematics subject Classification 2000: 35K05, 35K99, 47J06, 47H10

\section{Introduction}

Throughout this paper, $\mathcal{H}$ will denote a Hilbert space endowed with the inner product $\langle\cdot, \cdot\rangle$ and the norm $\|\cdot\|$. Let $T$ be a positive number and $\Omega$ be an open, bounded and connected domain in $\Omega \in \mathbb{R}^{d}(d \geq 1)$ with a smooth boundary $\partial \Omega$. We consider the problem of finding a function $u:[0, T] \rightarrow \mathcal{H}$ satisfes the initial boundary value problem for a class of fourth order strongly damped linear wave equations

$$
u_{t t}-\Delta u+\Delta^{2} u-\gamma \Delta u_{t}=\mathcal{R}(x, t), \quad(x, t) \in \Omega \times[0, T],
$$

subject to the conditions

$$
\begin{cases}u=\varphi, \quad u_{t}=\chi, & (x, t) \in \Omega \times\{T\}, \\ u=\Delta u=0, & (x, t) \in \partial \Omega \times[0, T] .\end{cases}
$$

There exists a sequence of positive real eigenvalues of the operator $-\Delta$, which denoted by $\left\{\lambda_{k}\right\}_{k \geq 1}$ where

$$
\left\{\begin{array}{l}
0<\lambda_{1} \leq \lambda_{2} \leq \lambda_{3} \leq \ldots \leq \lambda_{k} \leq \ldots, \\
\lambda_{k} \rightarrow \infty \text { as } k \rightarrow \infty
\end{array}\right.
$$

\footnotetext{
*e-mail: namdhq@tdmu.edu.vn

**e-mail: nguyenhuucan@tdtu.edu.vn (corresponding author)

***e-mail: vvau@ctuet.edu.vn

****e-mail: tranthanhbinh@sgu.edu.vn
} 
Moreover, there exists an orthonormal basis $\left\{\xi_{k}\right\}_{k \geq 1}$, where $\xi_{k}$ is an eigenfunction corresponding to $\lambda_{k}$

$$
\begin{cases}-\Delta \xi_{k}(x)=\lambda_{k} \xi_{k}(x), & x \in \Omega \\ \xi_{k}(x)=0, & x \in \partial \Omega\end{cases}
$$

And $\gamma>2$ is a positive constant, satisfies $\left(\gamma^{2}-4\right) \lambda_{1}-4>0$.

In practice, the data $(\varphi, \chi) \in \mathcal{H} \times \mathcal{H}$ is obtained by measurement contaminated with noise. Hence, instead of $(\varphi, \chi)$, we have the observation data $\left(\varphi^{\alpha}, \chi^{\alpha}\right)$ satisfying

$$
\left\|\varphi-\varphi^{\alpha}\right\|+\left\|\chi-\chi^{\alpha}\right\| \leq \alpha
$$

where the constant $\alpha>0$ represents a bound on the measurement error.

In the last years, the class of fourth order strongly damped wave equations has been investigated by many researchers, for example, existence, global classical solution, attractor, well-posedness, decay estimates, blowup, controllability, bootstrapping (e.g., [3]-[10]). Another important aspect of the qualitative study for the solutions of strongly damped wave equations is ill-posedness (in the sense of Hadamard).

In 2013, Xu Runzhang and Yanbing Yang [11] considered the global existence and asymptotic behaviour of solutions for a class of fourth order strongly damped nonlinear wave equations but they did not regularize it. Our work provide a regularization method for the solution of the ill-posed problem (1.1) - (1.2).

There are three sections in this paper. In Section 1, we have introduced our problem. The exact solution and ill-posedness of the inverse problem (1.1) - (1.2) are given in Section 2. Moreover, in Subsection 2.2, we give an example to show that the problem (1.1) - (1.2) is not stable. In Section 3, we develop a regularization method based on the truncated Fourier method and the stability estimate is established.

\section{Exact solution and ill-posedness of the inverse problem}

\subsection{Exact solution}

Assume that the problem (1.1) - (1.2) has a unique solution in the series form

$$
u(x, t)=\sum_{k=1}^{\infty}\left\langle u, \xi_{k}\right\rangle \xi_{k} .
$$

Then $u(x, t)$ satisfies the following system

$$
\left\{\begin{array}{l}
\frac{d^{2}}{d t^{2}} u_{k}(t)+\gamma \lambda_{k} \frac{d}{d t} u_{k}(t)+\left(\lambda_{k}+\lambda_{k}^{2}\right) u_{k}(t)=\mathcal{R}_{k}(t), \quad t \in(0, T), \\
u_{k}(T)=\varphi_{k}, \quad \frac{d}{d t} u_{k}(T)=\chi_{k},
\end{array}\right.
$$

where $u_{k}=\left\langle u, \xi_{k}\right\rangle, \varphi_{k}=\left\langle\varphi, \xi_{k}\right\rangle, \chi_{k}=\left\langle\chi, \xi_{k}\right\rangle, \mathcal{R}_{k}=\left\langle\mathcal{R}, \xi_{k}\right\rangle$.

For any $\gamma>2$ satisfies $\left(\gamma^{2}-4\right) \lambda_{1}-4>0$, the solution of (2.7) is given by:

$$
\begin{array}{r}
u_{k}(t)=\frac{\mu_{k}^{+} \exp \left(\mu_{k}^{-}(T-t)\right)-\mu_{k}^{-} \exp \left(\mu_{k}^{+}(T-t)\right)}{\mu_{k}^{+}-\mu_{k}^{-}} \varphi_{k}-\frac{\exp \left(\mu_{k}^{+}(T-t)\right)-\exp \left(\mu_{k}^{-}(T-t)\right)}{\mu_{k}^{+}-\mu_{k}^{-}} \chi_{k} \\
+\int_{t}^{T} \frac{\exp \left(\mu_{k}^{+}(s-t)\right)-\exp \left(\mu_{k}^{-}(s-t)\right)}{\mu_{k}^{+}-\mu_{k}^{-}} \mathcal{R}_{k}(s) d s,
\end{array}
$$




\section{ICM 2018}

where we define

$$
\mu_{k}^{+}=\frac{1}{2}\left(\gamma \lambda_{k}+\delta_{k}^{\frac{1}{2}}\right), \quad \mu_{k}^{-}=\frac{1}{2}\left(\gamma \lambda_{k}-\delta_{k}^{\frac{1}{2}}\right), \quad \delta_{k}=\left(\gamma^{2}-4\right) \lambda_{k}^{2}-4 \lambda_{k} .
$$

Let us define the operators $\mathbb{G}(t)$ and $\mathbb{K}(t)$ as follow

$$
\mathbb{G}(t) w=\sum_{k=1}^{\infty} \frac{\mu_{k}^{+} \exp \left(\mu_{k}^{-} t\right)-\mu_{k}^{-} \exp \left(\mu_{k}^{+} t\right)}{\mu_{k}^{+}-\mu_{k}^{-}}\left\langle w, \xi_{k}\right\rangle \xi_{k},
$$

and

$$
\mathbb{K}(t) w=\sum_{k=1}^{\infty} \frac{\exp \left(\mu_{k}^{-} t\right)-\exp \left(\mu_{k}^{+} t\right)}{\mu_{k}^{+}-\mu_{k}^{-}}\left\langle w, \xi_{k}\right\rangle \xi_{k},
$$

for $w \in \mathcal{H}$. From above observations, we can rewrite (2.8) as

$$
u(x, t)=\mathbb{G}(T-t) \varphi-\mathbb{K}(T-t) \chi+\int_{t}^{T} \mathbb{K}(s-t) \mathcal{R}(s) d s
$$

\subsection{III-posedness of the inverse problem}

In this subsection, we give an example which shows that the solution $u^{*}(x, t)$ of problem $(1.1)-(1.2)$ (if it exists) is not stable.

- Let $\varphi^{*}, \chi^{*}$, and $\mathcal{R}^{*}$ be defined as follows

$$
u^{*}(x, T)=\varphi^{*}(x)=0, \quad \partial_{t} u^{*}(x, T)=\chi^{*}(x)=\frac{\xi_{m}(x)}{\sqrt{\lambda_{m}}}, \quad \mathcal{R}^{*}=\sum_{m=1}^{\infty} \frac{2 \exp \left(-\gamma T \lambda_{m}\right)}{T^{3}}
$$

Then $u^{*}$ satisfy the integral equation

$$
u^{*}(x, t)=-\mathbb{K}(T-t) \chi^{*}+\int_{t}^{T} \mathbb{K}(s-t) \mathcal{R}^{*}(s) d s .
$$

We have

$$
\begin{aligned}
\left\|\mathbb{K}(T-t) \chi^{*}\right\|^{2} & =\left(\frac{\left.\exp \left(\mu_{m}^{-}(T-t)\right)-\exp \mu_{m}^{+}(T-t)\right)}{\mu_{m}^{+}-\mu_{m}^{-}}\right)^{2} \frac{1}{\lambda_{m}} \\
& =\frac{\exp \left(2 \mu_{m}^{+}(T-t)\right)\left(1-\exp \left(-(T-t) \sqrt{\delta_{m}}\right)\right)}{\lambda_{m} \delta_{m}}
\end{aligned}
$$

where $\delta_{m}=\left(\gamma^{2}-4\right) \lambda_{m}^{2}-4 \lambda_{m}$, noting that $\mu_{m}^{+}-\mu_{m}^{-}=\sqrt{\delta_{m}}$.

This leads to

$$
\left\|\mathbb{K}(T-t) \chi^{*}\right\|^{2}=\frac{\exp \left(2 \mu_{m}^{+}(T-t)\right)\left(1-\exp \left(-(T-t) \sqrt{\left(\gamma^{2}-4\right) \lambda_{m}^{2}-4 \lambda_{m}}\right)\right)^{2}}{\lambda_{m}\left[\left(\gamma^{2}-4\right) \lambda_{m}^{2}-4 \lambda_{m}\right]}
$$


It is easy to see that the function $f(t)=\exp \left(\mu_{m}^{+}(T-t)\right)\left(1-\exp \left(-(T-t) \sqrt{\left(\gamma^{2}-4\right) \lambda_{1}^{2}-4 \lambda_{1}}\right)\right)$ is a decreasing function for $t \in(0, T)$, we deduce that

$$
\begin{aligned}
\sup _{0 \leq t \leq T}\left\|\mathbb{K}(T-t) \chi^{*}\right\| & \geq \sup _{0 \leq t \leq T} \frac{\exp \left(\mu_{m}^{+}(T-t)\right)\left(1-\exp \left(-(T-t) \sqrt{\left(\gamma^{2}-4\right) \lambda_{1}^{2}-4 \lambda_{1}}\right)\right)}{\sqrt{\lambda_{m}\left[\left(\gamma^{2}-4\right) \lambda_{m}^{2}-4 \lambda_{m}\right]}} \\
& =\frac{\exp \left(\mu_{m}^{+} T\right)\left(1-\exp \left(-T \sqrt{\left(\gamma^{2}-4\right) \lambda_{1}^{2}-4 \lambda_{1}}\right)\right)}{\sqrt{\lambda_{m}\left[\left(\gamma^{2}-4\right) \lambda_{m}^{2}-4 \lambda_{m}\right]}} \\
& \geq \frac{\exp \left(\frac{\gamma \lambda_{m}}{2} T\right)\left(1-\exp \left(-T \sqrt{\left(\gamma^{2}-4\right) \lambda_{1}^{2}-4 \lambda_{1}}\right)\right)}{\sqrt{\lambda_{m}\left[\left(\gamma^{2}-4\right) \lambda_{m}^{2}-4 \lambda_{m}\right]}} .
\end{aligned}
$$

On the other hand, we get

$$
\begin{aligned}
& \left\|\int_{t}^{T} \mathbb{K}(s-t) \mathcal{R}^{*} d s\right\| \leq \int_{t}^{T} \sqrt{\sum_{m=1}^{\infty}\left[\frac{\exp \left(\mu_{m}^{-}(s-t)\right)-\exp \left(\mu_{m}^{+}(s-t)\right)}{\mu_{m}^{+}-\mu_{m}^{-}}\right]^{2} \frac{4 \exp \left(-2 \gamma T \lambda_{m}\right)}{T^{6}}} d s \\
& =\int_{t}^{T} \sqrt{\sum_{m=1}^{\infty} \exp \left(2(s-t)\left(\mu_{m}^{+}+\mu_{m}^{-}\right)\right)\left[\frac{\exp \left(-\mu_{m}^{+}(s-t)\right)-\exp \left(-\mu_{m}^{-}(s-t)\right)}{\mu_{m}^{+}-\mu_{m}^{-}}\right]^{2} \frac{4 \exp \left(-2 \gamma T \lambda_{m}\right)}{T^{6}}} d s .
\end{aligned}
$$

Using the inequality $|\exp (-y)-\exp (-z)| \leq|y-z|$, for $y, z>0$ and noting that $\mu_{m}^{+}+\mu_{m}^{+}=\gamma \lambda_{m}$, we obtain

$$
\begin{aligned}
\left\|\int_{t}^{T} \mathbb{K}(s-t) \mathcal{R}^{*} d s\right\| & \leq \int_{t}^{T} \sqrt{\sum_{m=1}^{\infty} \exp \left(2 \gamma \lambda_{m}(s-t)\right)(s-t)^{2} \frac{4 \exp \left(-2 \gamma T \lambda_{m}\right)}{T^{6}}} d s \\
& \leq \int_{t}^{T} \frac{2(s-t)}{T^{3}} d s \leq \frac{1}{T}
\end{aligned}
$$

Combining (2.15) and (2.16), we have

$$
\begin{aligned}
\left\|u^{*}\right\| & \geq\left\|\mathbb{K}(T-t) \chi^{*}\right\|-\left\|\int_{t}^{T} \mathbb{K}(s-t) \mathcal{R}^{*}(s) d s\right\| \\
& \geq \frac{\exp \left(\frac{\gamma \lambda_{m}}{2} T\right)\left(1-\exp \left(-T \sqrt{\left(\gamma^{2}-4\right) \lambda_{1}^{2}-4 \lambda_{1}}\right)\right)}{\sqrt{\lambda_{m}\left[\left(\gamma^{2}-4\right) \lambda_{m}^{2}-4 \lambda_{m}\right]}}-\frac{1}{T} .
\end{aligned}
$$

As $m \rightarrow \infty$, we get

$$
\begin{gathered}
\lim _{m \rightarrow \infty}\left(\left\|\varphi_{m}\right\|+\left\|\chi_{m}\right\|\right)=\lim _{m \rightarrow \infty} \frac{1}{\sqrt{\lambda_{m}}}=0 . \\
\lim _{m \rightarrow \infty}\left\|u^{*}\right\|_{C([0, T] ; \mathcal{H})}=\lim _{m \rightarrow \infty}\left(\frac{\exp \left(\frac{\gamma \lambda_{m}}{2} T\right)\left(1-\exp \left(-T \sqrt{\left.\left(\gamma^{2}-4\right) \lambda_{1}^{2}-4 \lambda_{1}\right)}\right)\right.}{\sqrt{\lambda_{m}\left[\left(\gamma^{2}-4\right) \lambda_{m}^{2}-4 \lambda_{m}\right]}}-\frac{1}{T}\right)=\infty .
\end{gathered}
$$

- Let $\varphi^{* *}=\chi^{* *}=\mathcal{R}^{* *}=0$, we deduce $u^{* *}=0$. This shows that a small perturbation of a physical measurement makes the corresponding solution have a large error. Thus the problem (1.1)-(1.2) is ill-posed in the sense of Hadamard.

Next section, we propose a regularization method for stabilizing the problem. 


\section{Fourier's truncation method}

Let $N=\left\{k \in \mathbb{N}\right.$ such that $k \geq 1$ and $\left.\lambda_{k} \leq \Lambda(\alpha)\right\}$. Without loss of generality, we assume that $\Lambda(\alpha)$ be a regularization parameter satisfying

$$
\lim _{\alpha \rightarrow 0} \Lambda(\alpha)=\infty
$$

For $w \in \mathcal{H}$, let us define the truncated version of (2.10) and (2.11) as

$$
\mathbb{G}_{\Lambda(\alpha)}(t) w=\sum_{k \in N} \frac{\mu_{k}^{+} \exp \left(\mu_{k}^{-} t\right)-\mu_{k}^{-} \exp \left(\mu_{k}^{+} t\right)}{\mu_{k}^{+}-\mu_{k}^{-}}\left\langle w, \xi_{k}\right\rangle \xi_{k}
$$

and

$$
\mathbb{K}_{\Lambda(\alpha)}(t) w=\sum_{k \in N} \frac{\exp \left(\mu_{k}^{-} t\right)-\exp \left(\mu_{k}^{+} t\right)}{\mu_{k}^{+}-\mu_{k}^{-}}\left\langle w, \xi_{k}\right\rangle \xi_{k} .
$$

Note that we pick $\Lambda(\alpha)$ so large that $\mathbb{G}, \mathbb{K}$ are retained.

Let us define the regularized solution by Fourier's truncation method as follows

$$
u^{\Lambda(\alpha)}=\mathbb{G}_{\Lambda(\alpha)}(T-t) \varphi^{\alpha}-\mathbb{K}_{\Lambda(\alpha)}(T-t) \chi^{\alpha}+\int_{t}^{T} \mathbb{K}_{\Lambda(\alpha)}(\omega-t) \mathcal{R}(x, \omega) d \omega .
$$

We have the following lemma which shall be useful in next results.

Lemma 3.1 For $t \in[0, T]$, we have

$$
\begin{aligned}
& \left\|\mathbb{G}_{\Lambda(\alpha)}(t)\right\|_{\mathcal{L}(\mathcal{H})} \leq \exp (t \gamma \Lambda(\alpha))\left(2+\frac{4 T}{\gamma^{2}}\right), \\
& \left\|\mathbb{K}_{\Lambda(\alpha)}(t)\right\|_{\mathcal{L}(\mathcal{H})} \leq \exp (t \gamma \Lambda(\alpha)) T
\end{aligned}
$$

Proof.

- From (2.9) and (3.19), let $w \in \mathcal{H}$, we have

$$
\begin{aligned}
\left\|\mathbb{G}_{\Lambda(\alpha)}(t) w\right\|^{2} & =\sum_{k \in N}\left[\frac{\mu_{k}^{+} \exp \left(\mu_{k}^{-} t\right)-\mu_{k}^{-} \exp \left(\mu_{k}^{+} t\right)}{\mu_{k}^{+}-\mu_{k}^{-}}\right]^{2}\left\langle w, \xi_{k}\right\rangle^{2} \\
& =\sum_{k \in N} \exp \left(2 t\left(\mu_{k}^{+}+\mu_{k}^{-}\right)\right)\left[\frac{\mu_{k}^{+} \exp \left(-t \mu_{k}^{+}\right)-\mu_{k}^{-} \exp \left(-t \mu_{k}^{-}\right)}{\mu_{k}^{+}-\mu_{k}^{-}}\right]^{2}\left\langle w, \xi_{k}\right\rangle^{2} \\
& \leq \sum_{k \in N} \exp (2 t \gamma \Lambda(\alpha))\left[\frac{\mu_{k}^{+} \exp \left(-t \mu_{k}^{+}\right)-\mu_{k}^{-} \exp \left(-t \mu_{k}^{-}\right)}{\sqrt{\delta_{k}}}\right]^{2}\left\langle w, \xi_{k}\right\rangle^{2},
\end{aligned}
$$

where $\mu_{k}^{-}+\mu_{k}^{-}=\gamma \lambda_{k}$ and $\mu_{k}^{+}-\mu_{k}^{-}=\sqrt{\delta_{k}}$.

On the other hand, using the inequality $(a+b)^{2} \leq 2\left(a^{2}+b^{2}\right)$ and $|\exp (-a)-\exp (-b)| \leq|a-b|$ for $a, b>0$, we have

$$
\begin{aligned}
\left(\frac{\mu_{k}^{+} \exp \left(-t \mu_{k}^{+}\right)-\mu_{k}^{-} \exp \left(-t \mu_{k}^{-}\right)}{\sqrt{\delta_{k}}}\right)^{2} & =\left(\exp \left(-t \mu_{k}^{+}\right)+\mu_{k}^{-} \frac{\exp \left(-t \mu_{k}^{+}\right)-\exp \left(-t \mu_{k}^{-}\right)}{\sqrt{\delta_{k}}}\right)^{2} \\
& \leq 2 \exp \left(-2 t \mu_{k}^{+}\right)+2\left|\mu_{k}^{-}\right|^{2} t^{2} \\
& \leq 2+\left(\frac{2 \lambda_{k} t}{\gamma \lambda_{k}+\sqrt{\delta_{k}}}\right)^{2} \leq 2+\frac{4 T}{\gamma^{2}}
\end{aligned}
$$


Combining (3.22) and (3.23), we get

$$
\left\|\mathbb{G}_{\Lambda(\alpha)}(t) w\right\|^{2} \leq \exp (2 t \gamma \Lambda(\alpha))\left(2+\frac{4 T}{\gamma^{2}}\right)^{2}\|w\|^{2} .
$$

- From (2.9) and (3.20), let $w \in \mathcal{H}$, we have

$$
\begin{aligned}
\left\|\mathbb{K}_{\Lambda(\alpha)}(t) w\right\|^{2} & =\sum_{k \in N}\left(\frac{\exp \left(\mu_{k}^{-} t\right)-\exp \left(\mu_{k}^{+} t\right)}{\mu_{k}^{+}-\mu_{k}^{-}}\right)^{2}\left\langle w, \xi_{k}\right\rangle^{2} \\
& =\sum_{k \in N} \exp \left(2 t\left(\mu_{k}^{+}+\mu_{k}^{-}\right)\right)\left(\frac{\exp \left(-t \mu_{k}^{+}\right)-\exp \left(-t \mu_{k}^{-}\right)}{\mu_{k}^{+}-\mu_{k}^{-}}\right)^{2}\left\langle w, \xi_{k}\right\rangle^{2} \\
& \leq \sum_{k \in N} \exp (2 t \gamma \Lambda(\alpha)) T^{2}\left\langle w, \xi_{k}\right\rangle^{2}=\exp (2 t \gamma \Lambda(\alpha)) T^{2}\|w\|^{2}
\end{aligned}
$$

This completes the proof of Lemma 3.1.

Before we formulate the main theorem, we also introduce the Gevrey class of functions of order $p>0$ and index $r>0$, see e.g. [1], defined by the spectrum of the Laplacian is denoted by

$$
\mathbf{B}_{p, r}:=\left\{f \in L^{2}(\Omega): \sum_{k=1}^{\infty} \lambda_{k}^{2 p} \exp \left(2 r \lambda_{k}\right)\left\langle f, \xi_{k}(x)\right\rangle^{2}<\infty\right\},
$$

and their norms given by

$$
\|f\|_{\mathbf{B}_{p, r}}=\sqrt{\sum_{k=1}^{\infty} \lambda_{k}^{2 p} \exp \left(2 r \lambda_{k}\right)\left\langle f, \xi_{k}(x)\right\rangle^{2}} .
$$

Theorem 3.1 Let $\Lambda(\alpha)$ be a regularization parameter such that

$$
\Lambda(\alpha)>\frac{4}{\gamma^{2}-4}, \text { where } \lim _{\alpha \rightarrow 0} \Lambda(\alpha)=\infty \text { and } \lim _{\alpha \rightarrow 0} \exp (T \gamma \Lambda(\alpha)) \alpha=0 .
$$

Suppose that the problem (2.8) has a unique solution $u \in L^{\infty}\left(0, T ; \mathbf{B}_{\beta, \gamma T}\right)$ and the integral equation (3.21) has a unique solution $u^{\Lambda(\alpha)} \in C([0, T] ; \mathcal{H})$. Then the estimate between the exact solution and the regularized solution is given by

$$
\left\|u-u^{\Lambda(\alpha)}\right\| \leq \exp (t \gamma \Lambda(\alpha)) T_{\max } \alpha+\lambda_{N^{\alpha}}^{-\beta} \exp \left(-\gamma t \lambda_{N^{\alpha}}\right)\|u\|_{L^{\infty}\left(0, T ; \mathbf{B}_{\beta, \gamma T}\right)}
$$

where $T_{\max }=\max \left(2+\frac{4 T}{\gamma^{2}}, T\right), \quad N^{\alpha}$ is the integer part of $\Lambda(\alpha)$ and $\gamma>2$ is a positive constant, satisfies $\left(\gamma^{2}-4\right) \lambda_{1}-4>0$.

Proof.

First of all, we denote

$$
U^{\Lambda(\alpha)}(t)=\mathbb{G}_{\Lambda(\alpha)}(T-t) \varphi-\mathbb{K}_{\Lambda(\alpha)}(T-t) \chi+\int_{t}^{T} \mathbb{K}_{\Lambda(\alpha)}(\omega-t) \mathcal{R}(x, \omega) d \omega
$$

Estimating (3.28) is divided into 2 steps.

Step 1. Estimate $\left\|u^{\Lambda(\alpha)}(\cdot, t)-U^{\Lambda(\alpha)}(\cdot, t)\right\|$. 
Using (1.5), (3.21), (3.29) and Lemma 3.1, we have

$$
\begin{aligned}
\left\|u^{\Lambda(\alpha)}(\cdot, t)-U^{\Lambda(\alpha)}(\cdot, t)\right\| & \leq\left\|\mathbb{G}_{\Lambda(\alpha)}(T-t)\left(\varphi-\varphi^{\alpha}\right)\right\|+\left\|\mathbb{K}_{\Lambda(\alpha)}(T-t)\left(\chi-\chi^{\alpha}\right)\right\| \\
& \leq \exp (t \gamma \Lambda(\alpha))\left(2+\frac{4 T}{\gamma^{2}}\right)\left(\varphi-\varphi^{\alpha}\right)+\exp (t \gamma \Lambda(\alpha)) T\left(\chi-\chi^{\alpha}\right) \\
& \leq \exp (t \gamma \Lambda(\alpha)) T_{\max } \alpha
\end{aligned}
$$

where $T_{\max }=\max \left(2+\frac{4 T}{\gamma^{2}}, T\right)$.

Step 2. Estimate $\left\|u(\cdot, t)-U^{\Lambda(\alpha)}(\cdot, t)\right\|$.

It is easy to see that

$$
\sum_{k=1}^{N^{\alpha}} u_{k}(t) \xi_{k}=\mathbb{G}_{\Lambda(\alpha)}(T-t) \varphi+\mathbb{K}_{\Lambda(\alpha)}(T-t) \chi-\int_{t}^{T} \mathbb{K}_{\Lambda(\alpha)}(\omega-t) \mathcal{R}(x, \omega) \mathrm{d} \omega .
$$

Therefore, using Lemma 3.1, we obtain

$$
\begin{aligned}
\left\|u(\cdot, t)-U^{\Lambda(\alpha)}(\cdot, t)\right\| & \leq\left\|u(\cdot, t)-\sum_{k=1}^{N^{\alpha}} u_{k}(t) \xi_{k}\right\|+\left\|\sum_{k=1}^{N^{\alpha}} u_{k}(t) \xi_{k}-U^{\Lambda(\alpha)}(\cdot, t)\right\| \\
& \leq \sqrt{\sum_{k=N^{\alpha}+1}^{\infty} \lambda_{N^{\alpha}}^{-2 \beta} \exp \left(-2 \gamma t \lambda_{N^{\alpha}}\right) \lambda_{k}^{2 \beta} \exp \left(2 \gamma t \lambda_{k}\right)\left|u_{k}(t)\right|^{2}} \\
& \leq \lambda_{N^{\alpha}}^{-\beta} \exp \left(-\gamma t \lambda_{N^{\alpha}}\right)\|u\|_{L^{\infty}\left(0, T ; \mathbf{B}_{\beta, \gamma T}\right)} .
\end{aligned}
$$

Thanks to triangle inequality $\left\|u-u^{\Lambda(\alpha)}\right\| \leq\left\|u-U^{\Lambda(\alpha)}\right\|+\left\|u^{\Lambda(\alpha)}-U^{\Lambda(\alpha)}\right\|$, combining (3.30) and (3.31) we have completed the proof of Theorem 3.1.

\section{References}

[1] C. Cao, M.A. Rammaha, and E.S. Titi, The Navier-Stokes equations on the rotating 2D sphere: Gevrey regularity and asymptotic degrees of freedom, Z. Angew. Math. Phys., 341-360 (1999).

[2] L. C. Evans, Partial Differential Equation, American Mathematical Society,Providence, Rhode Island, Volume 19 (1997).

[3] W. Fitzgibbon. Strongly damped quasilinear evolution equations, Journal of Mathematical Analysis and Applications, 79(2):536-550 (1981).

[4] V. Kalantarov and S. Zelik, Finite-dimensional attractors for the quasi-linear stronglydamped wave equation, Journal of Differential Equations, 247(4):1120-1155 (2009).

[5] S. Larsson, V. Thomée, and L. B. Wahlbin, Finite-element methods for a strongly damped wave equation, IMA journal of numerical analysis, 11(1):115-142 (1991).

[6] P. Massatt, Limiting behavior for strongly damped nonlinear wave equations, Journal of Differential Equations, 48(3):334-349 (1983).

[7] Nguyen Huy Tuan, Doan Vuong Nguyen, Vo Van Au, Daniel Lesnic, Recovering the initial distribution for strongly damped wave equation, Applied Mathematics Letters, 73: 69-77 (2017).

[8] V. Pata and M. Squassina, On the strongly damped wave equation, Communications in mathematical physics, 253(3):511-533 (2005). 
[9] V. Pata and S.V. Zelik, Smooth attractors for strongly damped wave equations, Nonlinearity, 1495-1506 (2006).

[10] V. Thomée and L. Wahlbin, Maximum-norm estimates for finite-element methods for a strongly damped wave equation, BIT Numerical Mathematics, 44(1):165-179 (2004).

[11] Xu Runzhang and Yanbing Yang, Global existence and asymptotic behaviour of solutions for a class of fourth order strongly damped nonlinear wave equations, Quarterly of Applied Mathematics , 71(3): 401-415 (2013). 